\title{
ÜBER DIE STRUCTUR DER DISCRIMINANTEN UND RESULTANTEN VON BINÄREN FORMEN ${ }^{1}$ \\ VON
}

\section{FRANZ MEYER}

in CLAUSTHAL.

I. Bricht man eine, nach steigenden Potenzen der Variabeln $\lambda$ geordnete binäre Form:

(I) $f_{m}(\lambda)=a_{0}+a_{1} \lambda+a_{2} \lambda^{2}+\ldots+a_{k-1} \lambda^{k-1}+a_{k} \lambda^{k}+a_{k+1} \lambda^{k+1}+\ldots+a_{m} \lambda^{m}$

hinter der $k^{\text {ten }}$ Potenz von $\lambda \mathrm{ab}$, so möge die so entstehende Form:

$$
\varphi_{k}(\lambda)=a_{0}+a_{1} \lambda+\ldots+a_{k} \lambda^{k}
$$

als die $) k^{\text {te }}$ Theilform v von $f$ bezeichnet werden, und entsprechend der nach Heraushebung des Factors $\lambda^{i}$ verbleibende Rest:

$$
\phi_{m-k}(\lambda)=a_{k}+a_{k+1} \lambda^{1}+\ldots+a_{m} \lambda^{m-k}
$$

als szugehörige Nebentheilform».

Gewisse Gründe sprechen dann dafür, dass in den Ausdruck für die Discriminante von $f$ die Discriminanten sämmtlicher Theilformen (2), (3) als Bestandtheile eingehen werden.

Die hiermit aufgeworfene Frage soll im Folgenden ihre Erledigung in bejahendem Sinne finden: ein analoges Resultat wird sich dann auch für die Resultante von zwei Binärformen angeben lassen. I und 2 .

$1 \mathrm{Vgl}$. die vorläufigen Mittheilungen in den Göttinger Nachrichten I $895, \mathrm{~N}^{\circ}$ 
Für zahlentheoretische Anwendungen dieser Ergebnisse hat man noch die Discriminante einer binären Form vermöge eines geeigneten Zahlenfactors zu )normiren».

Dabei wird sich zugleich die Frage beantworten lassen, was man überhaupt in der Zahlentheorie unter der Discriminante einer, ohne Binomialcoefficienten geschriebenen Binärform (I) zu verstehen hat.

2. In der Formentheorie legt man den Coefficienten $a_{0}, a_{1}, \ldots, a_{m-1}, a_{m}$ von (I) Gewichte bei von den Werthen $m, m-1, \ldots, \mathrm{I}, \mathrm{o}$; dadurch wird die Discriminante von (I), eine homogene Form der $a$ von der Ordnung $2(m-\mathrm{I})$, zugleich isobar d. i. alle Glieder der Discriminante erhalten das nämliche Gewicht $m(m-1)$.

Für unsern Zweck wird es sich indessen empfehlen, den $k$ ersten Coefficienten in (I) resp. die Gewichte $k, k-\mathrm{I}, \ldots, 2$, I beizulegen, allen übrigen aber das Gewicht Null.

Dann werden die einzelnen Glieder der Discriminante von (I) nicht alle das gleiche Gewicht besitzen, und es besteht die Vermuthung, dass gerade dem Aggregat der Glieder vom kleinsten Gewichte eine besonders einfache Eigenschaft zukommen wird.

Um zu dem gemeinten Aggregate zu gelangen, setze man in bekannter Weise: ${ }^{1}$

$$
a_{0}=\varepsilon^{k} a_{0}^{\prime}, \quad a_{1}=\varepsilon^{k-1} a_{1}^{\prime}, \ldots, a_{k-1}=\varepsilon a_{k-1}^{\prime}, \quad a_{k}=a_{k}^{\prime},
$$

entwickele die Discriminante nach steigenden Potenzen von $\varepsilon$, und ermittele den Coefficienten $C$ der kleinsten Potenz von $\approx$. Eben dies Product aus $C$ und der kleinsten Potenz von $\varepsilon$ liefert, wem man von den $a^{\prime}$ wiederum zu den $a$ zurückkehrt, das gewünschte Aggregat vom kleinsten Gewichte innerhalb der Discriminante.

3. Um den Coefficienten $C$ bequem zu berechnen, bedienen wir uns der Wurzeln von $f(\mathrm{I})$. Zu dem Behuf schicken wir folgenden Hülfssatz voraus:

Vermöge der Substitution (4) zerfallen die m Wurzeln von $f(\mathrm{I})$, als

1 Die Buchstaben $a^{\prime}$ in (4), wie die $\alpha$ und $\beta$ in (5) und (6) bedeuten Grössen, die nicht zugleich mit $\varepsilon$ versehwinden. 
Über die Structur der Discriminanten und Resultanten von binären Formen.

387

Functionen von $\varepsilon$ betrachtet, in zwei Klassen; die erste umfasst $k$ Wurzeln von der Form:

$$
\varepsilon \alpha_{1}+\varepsilon^{d} \beta_{1}, \ldots, \varepsilon \alpha_{k}+\varepsilon^{d} \beta_{k}, \quad(d>\mathrm{1}),^{1}
$$

die zweite die $m-k$ ïbrigen von der Form:

$$
\alpha_{k+1}+\varepsilon^{e} \beta_{k+1}, \ldots, \alpha_{m}+\varepsilon^{e} \beta_{m} \quad(e>0) .^{1}
$$

Hierbei sind die Grössen $\varepsilon \alpha$ in (5) die Wurzeln von $\varphi_{k}(2)$, und die Grössen $\alpha$ in (6) die Wurzeln von $\phi_{m-k}(3)$.

Dass die Wurzeln von $f$ die äussere Gestalt (5), (6) vermöge (4) erhalten, bedarf keines weiteren Beweises; auch, dass die $\alpha_{k+1}, \ldots, \alpha_{m}$ in (6) die Wurzeln von $\psi_{m-k}$ (3) sind, ergiebt sich fast unmittelbar, wenn man $\varepsilon \mathrm{zu}$ Null werden lässt, wodurch ja $f_{m}$ in $\lambda^{k} \phi_{m-k}$ übergehen würde.

Es handelt sich also nur noch um den Nachweis, dass die $\varepsilon \alpha_{1}, \ldots, \varepsilon \alpha_{k}$ in (5) die Wurzeln von $\varphi_{k}$ (2) sind.

Wir betrachten zu dem Ende die Quotienten $\frac{a_{0}}{a_{k}}, \frac{a_{1}}{a_{k}}, \ldots, \frac{a_{k-1}}{a_{k}}$. Mit Rücksicht auf (4), (5), (6) und die eben erledigte Bedeutung der $\alpha$ in (6) hat man zunächst:

$$
\begin{aligned}
(-1)^{k} \frac{a_{0}}{a_{k}}=\frac{(-1)^{m} \frac{\varepsilon^{k} a_{0}^{\prime}}{a_{m}}}{(-1)^{m-k} \frac{a_{k}}{a_{m}}} & =\frac{\left(\varepsilon \alpha_{1}+\varepsilon^{d} \beta_{1}\right) \ldots\left(\varepsilon \alpha_{k}+\varepsilon^{d} \beta_{k}\right)\left(\alpha_{k+1}+\varepsilon^{e} \beta_{k+1}\right) \ldots\left(\alpha_{m}+\varepsilon^{e} \beta_{m}\right)}{\alpha_{k+1} \ldots \alpha_{m}} \\
& =\varepsilon^{k}\left(\alpha_{1} \alpha_{2} \ldots \alpha_{k}\right)+\ldots
\end{aligned}
$$

wo die zuletzt rechterhand stehenden Punkte Glieder andeuten, die mit höheren Potenzen von $\varepsilon$ behaftet sind.

Da aber linkerhand nur die $k^{\text {te }}$ Potenz der willkürlichen Grösse $\varepsilon$ auftritt, so muss dasselbe auch rechts stattfinden $d$. h. es ist

$$
(-\mathrm{I})^{k} \frac{\varepsilon^{k} a_{0}^{\prime}}{a_{k}}=\left(\varepsilon \alpha_{1}\right)\left(\varepsilon \alpha_{2}\right) \ldots\left(\varepsilon \alpha_{k}\right)
$$

1 Um nicht in unnöthige Schwierigkeiten zu kommen, lassen wir es ganz dahin gestellt, welchen (ganzzahligen oder gebrochenen) Werth die später doch wieder herausfallenden Exponenten $d$ und $e$ in (5), (6) besitzen. 
Entsprechend verfährt man mit dem nächstfolgenden Quotienten $\frac{a_{1}}{a_{i}}$ :

$$
(-1)^{k-1} \frac{a_{1}}{a_{k}}=\frac{(-1)^{m-1} \frac{\varepsilon^{i-1} a_{1}^{\prime}}{a_{m}}}{(-1)^{m-k} \frac{a_{k}}{a_{m}}},
$$

mithin kommt für den Zähler des letzten Bruches:

$$
\begin{aligned}
& (-1)^{m-1} \frac{\varepsilon^{k-1} a_{1}^{\prime}}{a_{m}}\left(\alpha_{k+1} \ldots \alpha_{m}\right) \\
& =\left[\left(\varepsilon \alpha_{1}+\xi^{d} \beta_{1}\right) \ldots\left(\varepsilon \alpha_{k-1}+\varepsilon^{d} \beta_{k-1}\right)\left(\alpha_{k+1}+\xi^{p} \beta_{k+1}\right) \ldots\left(\alpha_{m}+\varepsilon^{p} \beta_{m}\right)\right. \\
& +\ldots \\
& +\quad \ldots \\
& \left.+\left(\varepsilon \alpha_{2}+\varepsilon^{\prime \prime} \beta_{n}\right) \ldots\left(\varepsilon \alpha_{k}+\varepsilon^{d} \beta_{k}\right)\left(\alpha_{k+1}+\varepsilon^{p} \beta_{k+1}\right) \ldots\left(\alpha_{m}+\varepsilon^{e} \beta_{m}\right)\right] \\
& +\left[\left(\varepsilon \alpha_{1}+\varepsilon^{i} \beta_{1}\right) \ldots\left(\varepsilon \alpha_{k}+\varepsilon^{i} \beta_{k}\right)\right)_{1}\left(\alpha_{k+1}+\varepsilon^{e} \beta_{k+1}\right) \ldots\left(\alpha_{m-1}+\varepsilon^{e} \beta_{m-1}\right)+\ldots \\
& \left.\left.+\left(\alpha_{k+2}+\xi^{e} \beta_{k+2}\right) \ldots\left(\alpha_{m}+\varepsilon^{e} \beta_{m}\right)\right\}\right] \text {. }
\end{aligned}
$$

Rechts muss sich wiederum Alles auf die $(k-1)^{\text {te }}$ Potenz von $\varepsilon$ reduciren, und es wird somit, nach beiderseitiger Division mit $\left(\alpha_{k+1} \ldots \alpha_{m}\right)$ :

$$
(-\mathrm{I})^{k-1} \frac{\varepsilon^{k-1} a_{1}^{\prime}}{a_{k}}=\left(s \alpha_{1}\right)\left(\varepsilon \alpha_{2}\right) \ldots\left(\Sigma \alpha_{k-1}\right)+\ldots+\left(\varepsilon \alpha_{2}\right)\left(\varepsilon \alpha_{3}\right) \ldots\left(\varepsilon \alpha_{k}\right)
$$

Fährt man so fort, so erkennt man aus (7), (8) und den sich anschliessenden Formeln sufort, dass die Grössen $\leqslant x_{1}, \varepsilon x_{2}, \ldots, \varepsilon x_{k}$ in (5) in der That mit den Wurzeln von $c_{k}$ (2) ïbereinstiminen.

Damit ist der zu Beginn dieser $N^{\circ}$ aufgestellte Hülfssatz ${ }^{1}$ bewiesen.

4. Die Discriminante $D_{m}$ einer binären Form $f_{m}$ (1) werde, bis auf einen, später zu normirenden Zahlenfactor $A_{m}$, definirt als das Quadrat des

1 Dieser Hülfssatz findet viclfache Auwendugy in der Theorie von Singularitaten von Curven, vel. die grosse Abhandlung des Verf. in den Wiener Monatsheften für Mathomatik und Physik I893. 
Über die Structur der Discriminanten und Resultanten von binären Formen. 389

Differenzenproductes der Wurzeln.von $f$, multiplicirt mit der $2(m-I)^{\text {ten }}$ Potenz des Coefficienten $a_{m}$ der höchsten Potenz von $\lambda$, in Zeichen:

(9) $D_{m}=A_{m} a_{m}^{2(m-1)} \Delta^{2}\left(\varepsilon \alpha_{1}+\varepsilon^{d} \beta_{1}, \ldots, \varepsilon \alpha_{k}+\varepsilon^{d} \beta_{k}, \alpha_{k+1}+\varepsilon^{e} \beta_{k+1}, \ldots, \alpha_{m}+\varepsilon^{e} \beta_{m}\right)$,

gemäss der Scheidung der Wurzeln von $f$ in die beiden Gruppen (5), (6). Eben vermöge dieser Scheidung zerfüllt das Quadrat des Differenzenproductes in drei wesentlich verschiedene Factoren:

$$
\begin{gathered}
\Delta^{2}\left(\varepsilon \alpha_{1}+\varepsilon^{d} \beta_{1}, \ldots, \varepsilon \alpha_{k}+\varepsilon^{d} \beta_{k}\right), \\
\Delta^{2}\left(\alpha_{k+1}+\varepsilon^{e} \beta_{k+1}, \ldots, \alpha_{m}+\varepsilon^{e} \beta_{m}\right),
\end{gathered}
$$

3) das Quadrat des Productes aus den Differenzen je einer Grösse (5) und je einer Grösse (6).

Für den Factor I) gilt aber:

$$
\text { I) } \begin{aligned}
\Delta^{2}\left(\varepsilon \alpha_{1}+\varepsilon^{d} \beta_{1}, \ldots, \varepsilon \alpha_{k}+\varepsilon^{d} \beta_{k}\right) & =\varepsilon^{k(k-1)} \Delta^{2}\left(\boldsymbol{\alpha}_{1}+\varepsilon^{d-1} \beta_{1}, \ldots, \alpha_{k}+\varepsilon^{d+1} \beta_{k}\right) \\
& =\varepsilon^{k(k-1)} \Delta^{2}\left(\boldsymbol{\alpha}_{1}, \boldsymbol{\alpha}_{2}, \ldots, \boldsymbol{\alpha}_{k}\right)+\ldots,
\end{aligned}
$$

wo die zuletzt stehenden Punkte wieder Glieder bezeichnen, die mit höheren Potenzen von $\varepsilon$ multiplicirt sind.

Entwickelt man andererseits die unter 2) und 3) aufgeführten Ausdrücke nach steigenden Potenzen von $\varepsilon$, so kommt:

$$
\begin{gathered}
\Delta^{2}\left(\alpha_{k+1}, \alpha_{k+2}, \ldots, \alpha_{m}\right)+\ldots, \\
\left(\alpha_{k+1} \alpha_{k+2} \ldots \alpha_{m}\right)^{2 k}+\ldots,
\end{gathered}
$$

wo es genügt, die (von $\varepsilon$ freien) Anfangsglieder zu notiren.

Somit liefert die Entwickelung der Discriminante $D_{m}$ (9) selbst nach steigenden Potenzen von $\varepsilon:^{1}$

$$
\left(9^{\prime}\right) \cdot D_{m}=\varepsilon^{k(k-1)} A_{m} a_{m}^{2(m-1)} \Delta^{2}\left(\alpha_{1}, \dot{\alpha}_{2}, \ldots, \alpha_{k}\right) \Delta^{2}\left(\alpha_{k+1}, \alpha_{k+2}, \ldots, \alpha_{m}\right)\left(\alpha_{k+1} \alpha_{k+2} \ldots \alpha_{m}\right)^{2 k}+\ldots
$$

Drückt man das hingeschriebene erste Glied von $D_{m}$ wieder rückwärts durch die ursprünglichen Coefficienten $a$ von $f$ aus, so hat man genau das Aggregat der Glieder in $D_{m}$, welche das Minimalgewicht, nemlich $k(k-\mathbf{I})$; besitzen.

1 Dass die Entwickelung von $D_{m}$ mit der $l(h-1)^{\text {ten }}$ Potenz von $\varepsilon$ beginnt, hätte, auch ohne irgend eine Kenntniss von der Gestalt der Wurzeln von $f$, aus der Bézout'schen Form der Discriminante $D_{m}$ geschlossen werden können. 
Bezeichnet man die Diseriminanten von $\varphi_{k}(2)$ und $\psi_{m-k}(3)$ mit $D_{k}$ resp. $D^{(m-k)}$, so ist, nach Analogie mit der Definition (9), und mit Rücksicht auf den Hülfssatz der $\mathrm{N}^{\circ} 3$ :

$$
\begin{aligned}
& D_{k}=A_{k} \cdot a_{k}^{2(k-1)} \Delta^{2}\left(\varepsilon \alpha_{1}, \varepsilon \alpha_{2}, \ldots, \varepsilon \alpha_{k}\right)=\varepsilon^{k(k-1)} A_{k} a_{k}^{\eta(k-1)} \Delta^{2}\left(\alpha_{1}, \alpha_{2}, \ldots, \alpha_{k}\right), \\
& D^{(m-k)}=A_{m-k} a^{2(m-k-1)} \Delta^{2}\left(\alpha_{k+1}, \alpha_{k+2}, \ldots, \alpha_{m}\right), \\
& \left(a_{k+1} a_{k+2} \ldots a_{m}\right)^{2}=\left(\frac{a_{k}}{a_{m}}\right)^{2 k} \text {. }
\end{aligned}
$$

Setzt man diese Werthe in das Anfangsglied (9') von $D_{m}$ ein, und beachtet, dass sich die Potenzen von $a_{m}$ in Zähler und Nenner zusammenziehen, wie folgt:

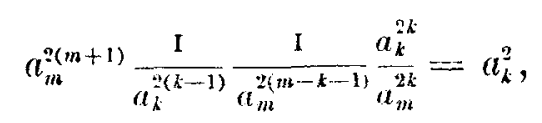

so ist das gemeinte Aggregat der Glieder in $D_{m}$ vom Minimalgewichte $k(k-1)$ identisch mit dem Producte:

$$
\frac{A_{n}}{A_{k} A_{m-k}} a_{k}^{2} D_{k} D^{(m-k)}
$$

Damit ist der Satz bewiesen:

Legt man den Coefficienten $a_{0}, a_{1}, \ldots, a_{k-1}, a_{k}, \ldots, a_{m}$ einer binüren Form $f_{m}(\lambda)$ :

$$
f_{m}(\lambda)=a_{0}+a_{1} \lambda+\ldots+a_{k-1} \lambda^{k-1}+a_{k} \lambda^{k}+\ldots+a_{m} \lambda^{m}
$$

successive die Gewichte $k, k-\mathbf{1}, \ldots, \mathbf{1}, 0, \ldots$, o bei, so zerfillt das Aggregat der Glieder in der Discriminante $D_{m}$ von $f$, welche das Mininalgewicht $k(k-1)$ besitzen, abgesehen von einem Zahlenfuctor, in die Factoren:

$$
a_{k}^{2} D_{k} D^{(m \cdot h)}
$$

wo $D_{k}, D^{(m-k)}$ die Discriminanten der Theilformen:

bedleuten.

$$
\left\{\begin{array}{c}
\varphi_{k}(\lambda)=a_{0}+a_{1} \lambda+\ldots+a_{k} \lambda^{k}, \\
c^{\prime \prime m-k}(\lambda)=a_{k}+a_{k+1} \lambda+\ldots+a_{m} \lambda^{m-k}
\end{array}\right.
$$


Ưber die Structur der Discriminanten und Resultanten von binären Formen.

391

Für $k=0$ (resp. $k=m$ ) wird der Satz bedeutungslos, während er für $k=\mathrm{I}$ (resp. $k=m-\mathrm{I})^{1}$ noch gültig bleibt, nur dass der Factor $D_{1}$ (resp. $D^{(1)}$ ) dann gar nicht auftritt, oder, was dasselbe ist, durch die Einheit zu ersetzen ist.

Symmetrischer hatte man den Coefficienten $a_{0}, a_{1}, \ldots, a_{k-1}, a_{k}, a_{k+1}, \ldots, a_{m}$ die Gewichte $k, k-1, \ldots, 1,0, \mathbf{1}, \ldots, m-k$ beilegen können; dann würde nur an Stelle des Minimalgewichts $k(k-1)$ unseres Satzes das Minimalgewicht $k(k-\mathrm{I})+(m-k)(m-k-\mathrm{I})$ treten.

5. Haben wir soeben den algebraischen Charakter des Ergebnisses hervortreten lassen, so möge nummehr unser Augenmerk auf die geeignete Bestimmung der in ( $\left.9^{\prime}\right)$ und (14) auftretenden Zahlenfactoren gerichtet sein.

Für den Augenblick denke man sich die Form $f_{m}(\lambda)(\mathbf{I})$ vermöge einer zweiten Variabeln $\mu$ homogen gemacht. Man bilde sodann nach der Sylvester'schen Vorschrift die Resultante $D_{m}^{\prime}$ der Formen $\frac{\partial f}{\partial \lambda}$ und $\frac{\partial f}{\partial \mu}$, also

$$
D_{m}^{\prime}
$$

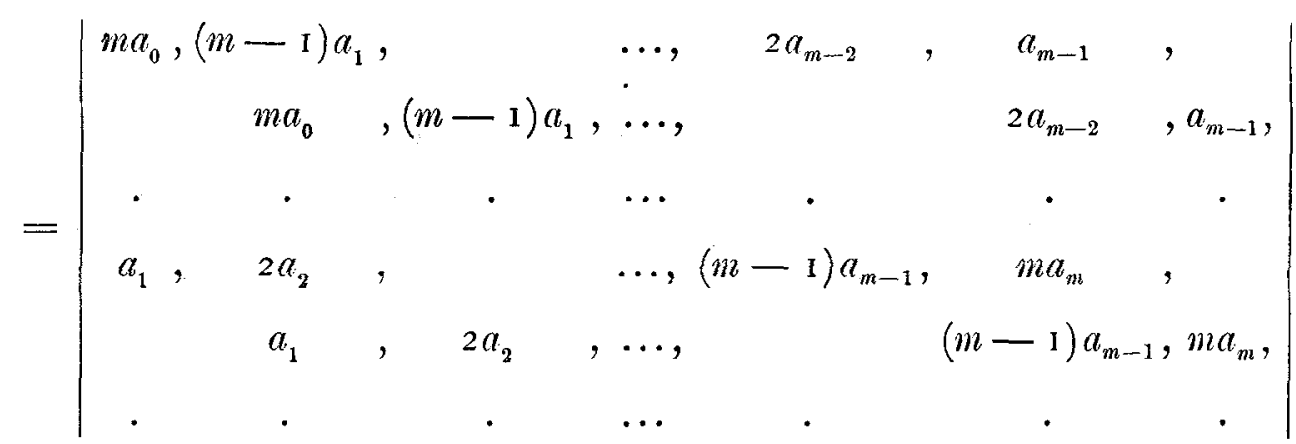

Nennen wir die Wurzeln von $f$ kurz $A_{1}, A_{2}, \ldots, A_{n}$, so ist nach einer bekannten Formel $^{2}$ (Vgl. z. B. FÀ̇ dI BRUNo, l. c. p. 88 (I3)):

$$
D_{m}^{\prime}=(-\mathrm{I})^{\frac{1}{2} m(m-1)} m^{m-2} a_{m}^{2(m-1)} \Delta^{2}\left(A_{1}, A_{2}, \ldots, A_{m}\right) \text {. }
$$

${ }^{1}$ Der Fall $k=m-\mathbf{I}$ findet sich bei FÀ Dr Bruvo, Theorie der binären Formen, Deutsche Ausgabe, p. 90 unten.

2 Die gemeinte Formel ist daselbst für die Resultante von $\frac{1}{m} \frac{\partial f}{\partial \lambda}$ und $\frac{1}{m} \frac{\partial f}{\partial \mu}$ aufgestellt, nach leichter Umrechnung geht daraus die Formel (I6) das Textes hervor. 
Nun ist $\Delta^{2}\left(A_{1}, A_{2}, \ldots, A_{m}\right)$, als ganzahlige symmetrische Function der $A$, eine ganzzahlige ganzrationale Function der elementar-symmetrischen Functionen der $A$, und zwar vom Grade $2(m-\mathrm{I})$. Also ist das Product $a_{m}^{2(m-1)} \Delta^{2}\left(A_{1}, A_{2}, \ldots, A_{n}\right)$ eine ganzzahlige homogene Form der Coefficienten $a$. $\mathrm{Da}$ das Gleiche auch von der linken Seite $D_{m}^{\prime}$ der Formel (16) gilt, so muss $D_{m}^{\prime}$ durch $m^{m-2}$ theilbar ${ }^{1}$ sein. Vergleicht man dies Resultat mit der Definition $\left(9^{\prime}\right)$, so erkennt man, dass unan den Zahlenfactor $A_{m}$ am geeignetsten gleich $(-1)^{\frac{1}{2} m(m-1)}$ setzen wird:

$$
D_{m}=
$$

$(-\mathrm{I})^{\frac{1}{2} m(m-1)} a_{m}^{2_{(m-1)}} \Delta^{2}\left(\varepsilon \alpha_{1}+\varepsilon^{\prime \prime} \beta_{1}, \ldots, \Sigma_{k}+\varepsilon^{l} \beta_{k}, \alpha_{k+1}+\varepsilon^{e} \beta_{k+1}, \ldots, \alpha_{m}+\xi^{e} \beta_{m}\right)$.

Dann besteht zwischen $D_{m}$ und $D_{m}^{\prime}$ (15) der Zusammenhang:

$$
D_{m}=\frac{1}{m^{m-2}} D_{m}^{\prime}
$$

und $D_{m}$ ist ebenfalls eine ganzzahlige Form der $a$.

Damit reducirt sich der Zahlenfactor $\frac{A_{m}}{A_{k} \cdot A_{m-k}}$ in (I 4) auf

$$
\frac{(-\mathrm{I})^{\frac{1}{2} m(m-1)}}{(-\mathrm{I})^{\frac{1}{2}^{k}(k-1)}(-\mathrm{I})^{\frac{1}{\frac{1}{2}^{2}(m-k)(m-k-1)}}}=(-\mathrm{I})^{k(m-k)} .
$$

Auf Grund unseres Hauptsatzes können wir aber noch einen Schritt weiter gehen, und zeigen, dass die Form $D_{m}$ ( $\mathrm{I} z$ ) eine primitive ist $\mathrm{d}$. $\mathrm{h}$. dass der grösste gemeinschaftliche Theiler ihrer Coefficienten die Einheit ist.

Denn ware dieser Theiler von der Einheit verschieden, so müsste er anch in jeden Bestandtheile ron $D_{u}$ aufrehen. Setzt man aber $l i$ gleich $m-$ I, so ist, wie ( I 4 ) lehrt, $D_{m-1}$ ein solcher Bestandtheil. Dann müsste der gedachte Theiler auch in $D_{m-2}, D_{m-3}, \ldots, D_{2}$ aufgehen; es ist aber $D_{2}=4 a_{0} a_{2}-a_{1}^{2}$, also primitiv.

Wir drücken dieses Zwischenergebniss als einen besondern Satz aus:

Der grösste gemeinschaftliche Theiler aller Coefficienten der Discrimi-

1 Die Theilbarkeit von $D_{m}^{\prime}$ durch $m^{n-2}$ geht auch direct aus den Hauptsatze hervor, wenn man in (I4) $k=m$ - I setzt. 
Über die Structur der Diseriminanten und Resultanten von binären Formen. 393 nantendeterminante $D_{m}^{\prime}$ (15) ist gleich $m^{m-2}$, und demnach ist die durch (1 7) oder (9") definirte Discriminantenform $D_{m}=\frac{\mathrm{I}}{m^{m-2}} D_{m}^{\prime}$ primitiv.

Damit ist beiläufig die Frage entschieden, was man in der Zahlentheorie unter der Discriminante einer, ohne Binomialcoefficienten geschriebenen Binärform zu verstehen hat.

Auf Grund der Formel (1 8) sprechen wir folgende Ergänzung unseres Hauptsatzes ( $\left.\mathrm{N}^{\circ} 4\right)$ aus:

Der in dem Hauptsatze nicht berücksichtigte Zahlenfactor des Productes $a_{k}^{2} D_{k} D^{(m-k)}$ ist einfach gleich $(-\mathrm{I})^{k(m-k)}$, falls die Discriminante einer binären Form durch (1 7) definirt wird.

6. Einfacher gestalten sich die analogen Verhältnisse bei der Resultante zweier Binärformen:

$$
\left\{\begin{array}{l}
f_{m}(\lambda)=a_{0}+a_{1} \lambda+\ldots+a_{k} \lambda^{k}+a_{k+1} \lambda^{k+1}+\ldots+a_{m} \lambda^{m} \\
g_{n}(\lambda)=b_{0}+b_{1} \lambda+\ldots+b_{l} \lambda^{l}+b_{l+1} \lambda^{l+1}+\ldots+b_{n} \lambda^{n}
\end{array}\right.
$$

Indem wir hier wiederum mit der $k^{\text {ten }}$ resp. $l^{\text {ten }}$ Potenz von $\lambda$ abbrechen, erhalten wir die Theilformen:

$$
\left\{\begin{array}{l}
\varphi_{k}(\lambda)=a_{0}+a_{1} \lambda+\ldots+a_{k} \lambda^{k} \\
\varphi_{l}^{\prime}(\lambda)=b_{0}+b_{1} \lambda+\ldots+b_{l} \lambda^{l}
\end{array}\right.
$$

nebst den zugehörigen Nebentheilformen: V

$$
\left\{\begin{array}{l}
\psi_{m-k}(\lambda)=a_{k}+a_{k+1} \lambda+\ldots+a_{m} \lambda^{m-k} \\
\psi_{m-l}^{\prime}(\lambda)=b_{l}+b_{l+1} \lambda+\ldots+b_{n} \lambda^{n-l}
\end{array}\right.
$$

Unter der Resultante $R_{m n}$ von $f_{m}$ und $g_{n}$ verstehen wir; wie üblich, die offenbar primitive Form:

$$
R_{m n}=\left|\begin{array}{ccccc}
a_{0} & a_{1} & a_{2} & & \ldots \\
& a_{0} & a_{1} & a_{2} & \ldots \\
\cdot & . & . & . & \ldots \\
b_{0} & b_{1} & b_{2} & & \ldots \\
& b_{0} & b_{1} & b_{2} & \ldots \\
\cdot & . & . & . & \ldots
\end{array}\right| .
$$


Vermöge der Wurzeln $A_{1}, A_{2}, \ldots, A_{m}, B_{1}, B_{2}, \ldots, B_{n}$ von $f_{m}, g_{n}$ drückt sich $R_{m n}$ (Vgl. z. B. FAA dI Bruvo, l. c. p. 53 (12) und p. 74 oben) aus, wie folgt:

$$
\begin{array}{r}
R_{m n}=a_{m}^{n} b_{n}^{m}\left(A_{1}-B_{1}\right)\left(A_{2}-B_{1}\right) \ldots\left(A_{m}-B_{1}\right) \\
\left(A_{1}-B_{2}\right)\left(A_{2}-B_{2}\right) \ldots\left(A_{m}-B_{2}\right) \\
\ldots \\
. \\
\left(A_{1}-B_{n}\right)\left(A_{2}-B_{n}\right) \ldots\left(A_{m}-B_{n}\right) .
\end{array}
$$

Vermōge der Substitutionen:

$$
\left\{\begin{array}{lll}
a_{0}=\varepsilon^{k} a_{0}^{\prime}, & a_{1}=\varepsilon^{k-1} a_{1}^{\prime}, \ldots, a_{k-1}=\varepsilon a_{k-1}^{\prime}, & a_{k}=a_{k}^{\prime}, \\
b_{0}=\varepsilon^{\prime} b_{0}^{\prime}, & b_{1}=\varepsilon^{l-1} b_{1}^{\prime}, \ldots, b_{l-1}=\varepsilon b_{l-1}^{\prime}, & b_{l}=b_{l}^{\prime},
\end{array}\right.
$$

nehmen die Wurzeln $A, B$ die Gestalt an:

(25) $\begin{cases}\varepsilon \alpha_{1}+\varepsilon^{d} \beta_{1}, \ldots, \varepsilon \alpha_{k}+\varepsilon^{d} \beta_{k}, \alpha_{k+1}+\varepsilon^{e} \beta_{k+1}, \ldots, \alpha_{m}+\varepsilon^{e} \beta_{m} & (d>\mathrm{I}, e>0), \\ \varepsilon \alpha_{1}^{\prime}+\varepsilon^{d^{\prime}} \beta_{1}^{\prime}, \ldots, \varepsilon \alpha_{l}^{\prime}+\varepsilon^{d^{\prime}} \beta_{l}^{\prime}, \alpha_{l+1}^{\prime}+\varepsilon^{c^{\prime}} \beta_{l+1}^{\prime}, \ldots, \alpha_{n}^{\prime}+\varepsilon^{\prime} \beta_{n}^{\prime} & \left(d^{\prime}>\mathrm{I}, e^{\prime}>0\right) .\end{cases}$

Führt man nunmehr das Product (23) aus, so ergiebt sich durch eine ganz ähnliche Rechnung, wie in $\mathrm{N}^{\circ} 4$, dass das Aggregat der mit der niedrigsten, nemlich $(k l)^{\text {ten }}$ Potenz von $\cdot \varepsilon$ multiplicirten Glieder übereinstimmt mit dem Producte:

$$
(-\mathrm{I})^{l(m-k)} R_{k l} R^{(m-k, n-l)},
$$

wo $R_{k l}, R^{(m-k, n-l)}$ die Resultanten der Formen (20) resp. (2 I) sind.

Somit gilt der Satz:

Legt man den Coefficienten $a_{0}, a_{1}, \ldots, a_{k-1}, a_{k}, \ldots, a_{m} ; b_{0}, b_{1}, \ldots$, $b_{l-1}, b_{l}, \ldots, b_{n}$ der beiden binären Formen $f_{m}(\lambda), g_{m}(\lambda),(19)$ die Gewichte $k, k-\mathrm{I}, \ldots, \mathrm{I}, \mathrm{o}, 0 \ldots \circ$ resp. $l, l-\mathrm{I}, \ldots, \mathrm{I}, \mathrm{o}, 0, \ldots, 0$ bei, so zerfällt das Aggregat der Glieder in der Resultante $R_{m n}$ von $f$ und $g$, welche das Minimalgewicht kl besitzen, in das Product

$$
(-\mathrm{I})^{((m-k)} R_{k l} R^{(m-k, n-l)},
$$

wo $R_{k l}, R^{(m-k, n-l)}$ die Resultanten der Theilformen $\varphi_{k}(\lambda), \varphi_{i}^{\prime}(\lambda)(20)$ resp.

der Nebentheilformen $\psi_{m-k}(\lambda), \psi_{n-l}^{\prime}(\lambda)(2 \mathrm{I})$ bedeuten. 
Über die Structur der Discriminanten und Resultanten von binären Formen. 395

7. Die für die Discriminanten und Resultanten binärer Formen im Obigen aufgeworfenen und beantworteten Fragen lassen sich ohne Weiteres auch auf ternäre und höhere Formen ausdehnen; indessen stösst man hier auf solche Schwierigkeiten, dass eine Entscheidung vorderhand noch nicht möglich zu sein scheint.

Clausthal, d. 6 Februar I 895 . 